Elke Greifeneder, Adeline Krems und Maria Gäde Praxis adé? Der Mangel an Praxiseinblicken während der COVID-19-Pandemie Good-bye to practical training? The lack of insight into practice during the COVID-19 pandemic

https://doi.org/10.1515/bd-2021-0077

Zusammenfassung: In diesem Beitrag werden die Einschränkungen und Konsequenzen für Pflichtpraktika während der COVID-19 Pandemie thematisiert. Im Kontext der Praktikumsberatung des Instituts für Bibliotheks- und Informationswissenschaft der Humboldt-Universität zu Berlin wurden 81 Einrichtungen kontaktiert und angefragt, welche der Einrichtungen während der andauernden Einschränkungen ein Praktikumsangebot machen. Die Ergebnisse der Befragung, daraus resultierende Konsequenzen sowie Vorschläge für Ersatzleistungen oder Alternativen zum klassischen Praktikum (im digitalen Raum) werden mit den Vorund Nachteilen beschrieben.

Schlüsselwörter: Praktikum, Ausbildung, COVID-19-Pandemie, Bibliotheks- und Informationswissenschaft

Abstract: The paper investigates the impact of the pandemic and subsequent limitations on student internships required by study programmes. The internship advisory service of the Berlin School of Library and Information Science at Humboldt University contacted 81 institutions to ask whether they offered internships under corona restrictions. The results of this survey are presented here and suggestions for substitutes or digital alternatives to the traditional model of internship are outlined - with their advantages and disadvantages.

Keywords: Practical training, education, COVID-19 pandemic, library and information science

Prof. Dr. Elke Greifeneder: greifeneder@ibi.hu-berlin.de

Adeline Krems: adeline.krems@hu-berlin.de

Dr. Maria Gäde: maria.gaede@ibi.hu-berlin.de 


\section{Einleitung}

Die Pandemie stellt uns alle vor große Herausforderungen. Die Universitäten waren unter den ersten Einrichtungen, die komplett auf Online-Präsenz umgestellt haben und derzeit sieht es so aus, als wären die Studierenden auch mit die letzten, die dann wieder in die Institutsgebäude für Lehre vor Ort zurückkehren dürfen. In den letzten Monaten wurde intensiv über die Auswirkungen und Probleme der digitalen Lehre auf den Lernerfolg geforscht (Marczuk et al. 2021, Berghoff et al. 2021), ebenso über die finanziellen Sorgen der Studierenden, da ein Großteil der studentischen Jobs in Cafés, Restaurants oder Theatern weggefallen ist (Becker \& Lörz 2020). Wenig wurde über die Folgen der nicht angebotenen Praktikumsmöglichkeiten publiziert. Dieser Artikel schließt diese Lücke.

Nach einer kurzen Einführung in vergleichbare Studien stellen wir die Ergebnisse einer Studie vor, in der wir Praktikumseinrichtungen des Instituts für Bibliotheks- und Informationswissenschaft (IBI) an der Humboldt-Universität zu Berlin über die Möglichkeit, Praktika während der Pandemie durchzuführen, befragt haben.

\section{Praktika im Studium}

Alle bibliothekarischen Studiengänge in Deutschland fordern ein Pflichtpraktikum, wobei sich die Länge der Praktika zwischen Einrichtungen zwischen sieben Wochen und einem ganzen Semester unterscheidet. Praktika dienen dem Erwerb praktischer Kenntnisse über Aufgaben, Arbeitsabläufe und Methoden im bibliotheks- und Informationswissenschaftlichen Bereich sowie die Einübung sozialer Verhaltensnormen der Berufswelt. Praktika bieten die Gelegenheit, typische Tätigkeitsbereiche kennen zu lernen und dadurch auch noch gänzlich unbekannte und unerwartete Arbeitsbereiche für sich selbst zu entdecken. Nicht zuletzt besteht ein Zusammenhang zwischen geleisteten Praktika und einem späteren Berufseinstieg. So fand Sarcletti (2009) heraus, dass Praktika die Erfolgsquote bei Berufseinstieg erheblich steigern.

Es gibt wenige Berichte zur Situation von Praktika in Pandemiezeiten. Hering (2020) untersucht Stellenanzeigen für studentische Jobs und für Praktika auf der Plattform Indeed.com und kommt zum traurigen Ergebnis, dass der Höchststand in dem von ihr untersuchten Zeitraum der Juni 2020 war, in dem es 45 Prozent weniger Anzeigen für Praktika als im Vorjahr gab. Ferstl (2020) berichtet in einem Beitrag im Kurier über die Situation in Österreich und fasst prägnant zusammen, 
was der Mangel an Praktikumserfahrungen für Studierende bedeutet: „Kein Geld, keine Berufserfahrung, kein Abschluss“ (o.S.). Ähnlich wie am IBI kennt man durch die Prüfungsausschüsse, Studienberatungen und Sozialberatungen an den Universitäten viele Fälle, aber wie viele wirklich von einem abgesagten oder nicht möglichen Praktikum betroffen sind, wurde bis dato auch in Österreich nicht erhoben.

Am IBI müssen Studierende des Bachelor- und des Masterstudienganges ein siebenwöchiges Pflichtpraktikum in Vollzeit erbringen. Der Umfang des Praktikums einschließlich der Erarbeitung des Praktikumsberichtes beträgt 300 Stunden; ein Teilzeitpraktikum ist also möglich, solange die geforderte Stundenzahl erlangt wurde. Praktika sollen in der Regel in der vorlesungsfreien Zeit geleistet werden. Masterstudierende können anstatt eines Praktikums auch ein Projektmodul absolvieren. Diese Alternative empfiehlt die Studienberatung des IBI aber insbesondere Studierenden, die ausreichend praktische Erfahrungen durch frühere Praktika, studentische BID-Stellen oder eine FaMI-Ausbildung erlangt haben. Im Weiterbildenden Masterstudiengang Bibliotheks- und Informationswissenschaft gelten andere Regelungen für das Praktikum, doch auch sie müssen ein Praktikum leisten, wenn nicht ausreichend adäquate Berufserfahrung vorliegt.

\section{Praktikumssituation seit März 2020}

Die Autorinnen dieses Artikels sind alle Mitglieder im Prüfungsausschuss (PAU) des IBI. Im März 2020, als die erste Lockdown-Welle über Deutschland hereinbrach und gleichzeitig ungefähr Mitte der Semesterferien, häuften sich die Anfrage an den PAU, weil Praktika vor Ort kurzfristig abgesagt wurden und somit überhaupt nicht angetreten werden konnten oder begonnene Praktika plötzlich aufgrund der Situation beendet werden mussten. Gleichzeitig möchten wir betonen, wie viele Einrichtungen neben dem Stress der Situation trotzdem eine Weiterführung der Praktika im Homeoffice ermöglicht haben.

In den nächsten Semesterferien, im Sommer 2020, verschärfte sich die Situation, da die Möglichkeit digitaler Praktika die Ausnahme war und viele Studierende selbst für sich entschieden hatten, das Praktikum später zu leisten. In dieser Zeit stieg die Anzahl der Anträge für Ersatzleistungen für Praktika, zum Beispiel durch Projektseminare, sprunghaft an. Sowohl im Wintersemester 2020/21 als auch im Sommersemester 2021 bot das IBI daher drei anstatt regulär zweier Projektseminare an, um der gestiegenen Anzahl an Studierenden in den Kursen Rechnung zu tragen. Für Studierende, die alle anderen Leistungen bereits 
erbracht haben, ist es keine Option, viele Monate $\mathrm{zu}$ warten, um einen Praktikumsplatz nach der Pandemie zu bekommen.

Der nächste reguläre Zeitraum für Praktika, die Semesterferien zwischen Wintersemester 2020/2021 und Sommersemester 2021 brachte ein weiteres Problem mit sich: Die Berliner Senatskanzlei - Wissenschaft und Forschung hatte im Frühjahr 2020 beschlossen, dass das Wintersemester zwei Wochen später starten wird. Möglicherweise hatte man gehofft, dass sich die Pandemiesituation bis dahin verbessert hat und man auf ein zweites Digitalsemester hätte verzichten können. Diese Verschiebung des Beginns des Wintersemesters führte aber nun dazu, dass die Semesterpause zwischen den beiden Semestern nur noch sechs Wochen betrug. Siebenwöchige Pflichtpraktika wären also überhaupt nicht möglich gewesen. Um den Studierenden trotzdem ein Praktikum sowie eine kurze Pause zwischen den Semestern zu ermöglichen, einigte sich der Prüfungsausschuss auf eine einmalige Reduzierung des Pflichtpraktikums auf vier Wochen. Das Feedback der Studierenden und Praktikumseinrichtungen auf diese Maßnahme war jedoch verhalten, da ein verkürztes Praktikum eher unattraktiv für die Einrichtungen ist.

\section{Praktikumsangebote in der Pandemie}

Das IBI bietet zur Orientierung für Studierende, die nach einem Praktikumsplatz suchen, eine Liste von Praktikumseinrichtungen an, in denen zuvor erfolgreich Praktika durchgeführt wurden. Dabei handelt es sich um eine Auswahl von Bibliotheken, Verlagen, Archiven, Gedenkstätten und sonstigen Einrichtungen, die sich im weitesten Sinne mit der Verarbeitung und / oder Bereitstellung von Informationen beschäftigen. Um einen besseren Überblick zu erhalten, welche der Einrichtungen während der andauernden Einschränkungen überhaupt ein Praktikumsangebot machen, wurde vom 22. Februar bis 11. März 2021 eine umfassende Abfrage gestartet. Insgesamt wurden 81 Einrichtungen kontaktiert und befragt.

Besonders erfreulich ist die Rücklaufquote: Alle kontaktierten Einrichtungen antworteten auf die Anfragen. Die Ergebnisse der Befragung sind in der Abbildung dargestellt. Erwartungsgemäß hat die große Mehrheit der Einrichtungen (50) momentan keine Möglichkeit, ein Praktikum anzubieten - vier Einrichtungen bieten gar keine Praktika mehr an. Sehr zurückhaltend äußerte man sich außerdem zu zukünftigen Angeboten. Nur acht Einrichtungen planen fest, in der zweiten Jahreshälfte 2021 erneut Praktika durchzuführen. 13 Institutionen gaben an, je nach Verlauf der Pandemie zu entscheiden, ob und wann wieder Angebote 
gemacht werden können - eine Festlegung auf einen Zeitraum wurde nicht vorgenommen. Dies zeigt die große Unsicherheit und Vorsicht der Institutionen auch für die unmittelbare Zukunft. Lediglich sieben der 81 Institutionen bieten momentan regulär einen Praktikumsplatz an.

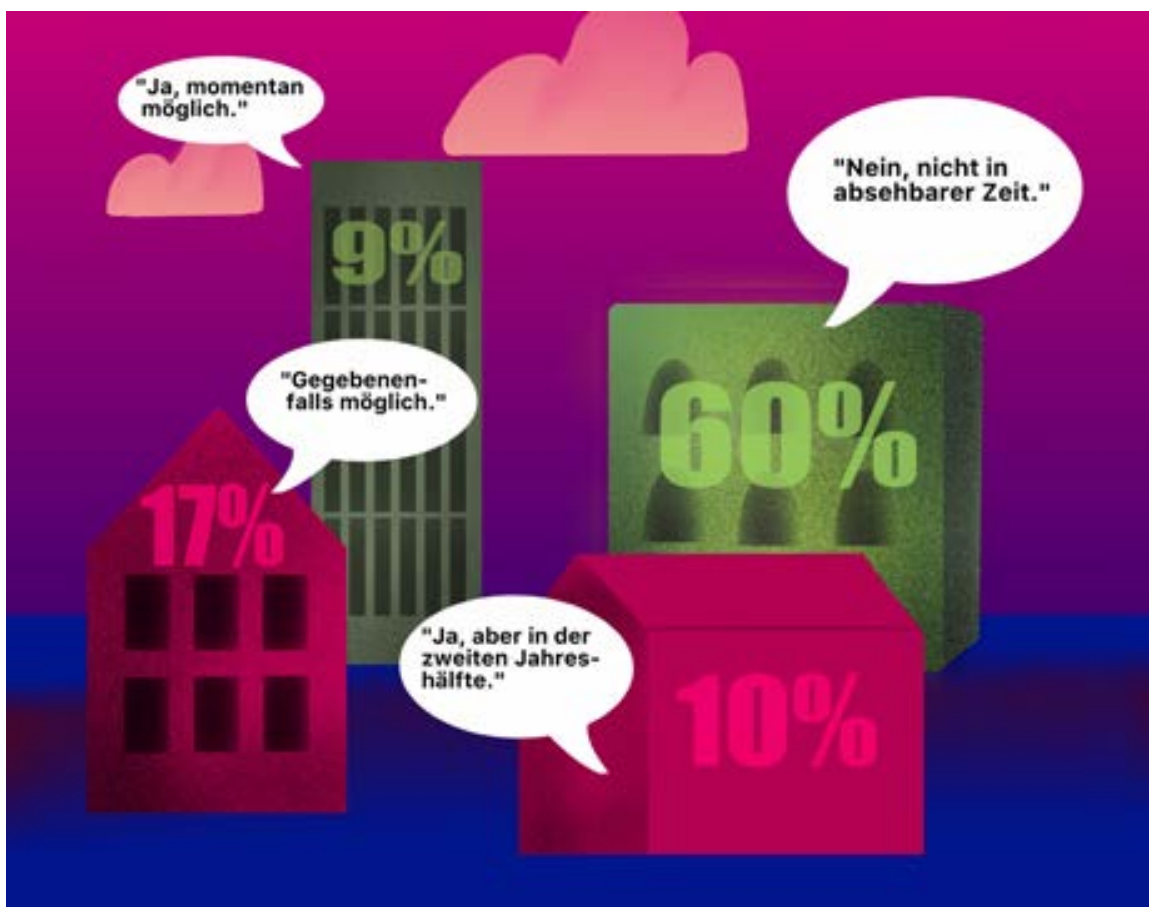

Abb.: Exemplarische Antworten zur Befragung von Praktikumsmöglichkeiten unter BID-Einrichtungen.

\section{Konsequenzen und Lösungsstrategien}

Der Mangel an Praktikumsangeboten und die unsichere Perspektive führen dazu, dass sich Studierende der Problematik von erheblichen Verzögerungen im Studienverlauf entgegensehen. Um dem entgegenzuwirken und die Studierbarkeit bzw. Studienabschlüsse weiterhin gewährleisten zu können, wurde gemeinsam an möglichen Alternativen und Ersatzleistungen gearbeitet. So wurde die erforderliche Praktikumszeit von sieben auf vier Wochen verkürzt. Damit sollte vor allem Studierenden geholfen werden, die in der Pandemie ein Praktikum begonnen hatten, dieses aber nicht mehr abschließen konnten. Als Ausgleich für diese ver- 
kürzte Praktikumszeit wurde eine Ersatzleistung festgelegt: Studierende, die das Praktikum nur in diesem verkürzten Umfang ableisten können, reichen zusätzlich zu den sonstigen erforderlichen Praktikumsunterlagen eine der folgenden schriftlichen Ersatzleistungen im Umfang von ca. 5 Seiten (max. $10.000 \mathrm{ZoL}^{1}$ ) ein, die individuell zu Hause erstellt werden kann:

- Eine ausführliche Beschreibung der Aufgaben und Dienstleistungen der Einrichtung, in der das Praktikum absolviert wurde ODER

- ein Kurzbericht zu einer Analyse der Barrierefreiheit der Website der Einrichtung, in der das Praktikum absolviert wurde ODER

- eine kurze SWOT-Analyse der Einrichtung, in der das Praktikum absolviert wurde.

Dabei ist völlig klar, dass ein vierwöchiges Praktikum weder für die Institutionen noch für die Studierenden eine zufriedenstellende Alternative darstellt. Für Praktika in der aktuellen Situation wurde zusätzlich beschlossen, dass Studierende in Abstimmung mit der Praktikumsinstitution Aufgaben teilweise oder vollständig im Homeoffice erledigen können. Einige Institutionen haben bereits von dieser Möglichkeit Gebrauch gemacht - erste Rückmeldungen zeigen positive Erfahrungen (siehe auch Kalová 2021). In manchen Fällen wurden so auch Praktika in entfernten Orten realisiert, die sonst aus finanziellen oder persönlichen Gründen nicht möglich gewesen wären (Kobert 2021). De Peralta (2020) berichtet über die Erfahrungen mit virtuellen Gruppenpraktika, in denen mehrere Studierende gemeinsam Projekte bearbeitet haben. Sie beschreibt, wie die Praktikant*innen durch diese neue Form viel mehr gelernt haben, auch in einem Praktikum eigenständig zu arbeiten, sich zu organisieren und Ergebnisse zu einer bestimmten Deadline liefern zu müssen. Ein ähnliches Konzept verfolgt die Data School Utrecht seit vielen Jahren erfolgreich mit ihrem Konzept des Embedded Research Assistant (Utrecht Data School 2021).

Ein Austausch mit dem OPL ${ }^{2}$-Arbeitskreis Berlin-Brandenburg zeigte eine große Bereitschaft und Offenheit gegenüber der Weiterentwicklung von Praktika im Zuge der Pandemie und der digitalen Transformation. Insbesondere One Person Libraries mit eingeschränkten Öffnungs- und Präsenzzeiten können von flexibleren Einsatzmöglichkeiten auch profitieren. Es wurde vereinbart, eine Liste potentieller Aufgaben zu erstellen, die für virtuelle Praktikumsformate in Frage kommen. Mit der fortschreitenden Digitalisierung in allen Bereichen ist zu erwarten, dass diese Liste von Aufgaben stetig wachsen kann. Entsprechend müssen

1 ZoL: Zeichen ohne Leerzeichen [Anmerkung der Redaktion].

2 OPL: One Person Library [Anmerkung der Redaktion]. 
Praktikumsrichtlinien und -vereinbarungen sich mit dem Konzept von virtuellen Praktika (im Englischen bekannter unter dem Begriff des „remote internships“) auseinandersetzen und entsprechende Regelungen treffen. Am IBI werden die Praktikumsrichtlinien nun auf die neue Situation hin überprüft und Anpassungen und Erweiterungen für das digitale Praktikum ergänzt.

Bei all dem sollte jedoch nicht vergessen werden, dass es auch Ziel des Praktikums ist, soziale Kompetenzen zu erlernen und Arbeitsalltage kennenzulernen. Nicht selten ist die Erfahrung im Praktikum ausschlaggebend für die spätere Berufswahl und kann erste Weichen setzen oder aber auch verdeutlichen, welche Wege nicht weiterverfolgt werden wollen. Sarcletti (2009, S. 237) konnte eine positive Korrelation zwischen der praktischen Erfahrung und dem Berufseinstieg nach dem Studium feststellen. Das Knüpfen von Kontakten und die Selbstpräsentation sind ein wesentlicher Teil des Praktikums. Diese soziale Komponente lässt sich nur bedingt in den digitalen Raum übertragen und sollte weiterhin als wichtiger Aspekt des Praktikums mitgedacht und wo möglich eine sinnvolle Kombination gefunden werden.

\section{Fazit}

Während es relativ schnell gelungen ist, die Online-Lehre in der Universität zu implementieren, fehlt es weiterhin an Angeboten und Lösungen für praktischen Anteile im Studium. Trotz der Bemühungen um Ersatzleistungen, Anerkennungen und die aktive Kommunikation mit Praktikumseinrichtungen ist für einige Studierende das Praktikum ein momentan unerreichbares Ziel. Es bleibt zu hoffen, dass diese fehlende Erfahrung für den Großteil der Studierenden nicht zum Nachteil wird und zukünftige Arbeitgeberinnen und Arbeitgeber die besondere Situation in Erinnerung behalten und auch denjenigen Studierenden eine Chance geben, denen bisher der praktische Einsatz verwehrt blieb.

\section{Referenzen}

Becker, Karsten; Lörz, Markus: Studieren während der Corona-Pandemie: Die finanzielle Situation von Studierenden und mögliche Auswirkungen auf das Studium. Hannover 2020, https://doi.org/10.34878/2020.09.dzhw_brief [Zugriff: 15.05.2021].

Berghoff, Sonja; Horstmann, Nina; Hüsch, Marc; Müller, Kathrin: Studium und Lehre in Zeiten der Corona-Pandemie: Die Sicht von Studierenden und Lehrenden. Gütersloh 2021, https://www.che.de/download/studium-lehre-corona [Zugriff: 16.05.2021]. 
Ferstl, Caroline: Kein Praktikum wegen Corona: Was das für Studierende bedeutet, https:// kurier.at/wirtschaft/kein-praktikum-wegen-corona-was-das-fuer-studierendebedeutet/400940108 [Zugriff: 16.05.2021].

Hering, Annina: Studi-Jobs und Praktika: Student:innen als große Verlierer:innen der CoronaKrise, https://www.hiringlab.org/de/blog/2020/10/22/corona-krise-studenten [Zugriff: 16.05.2021].

Kalová, Tereza: Bibliothekspraktikum während der COVID-19-Pandemie: Erfahrungen mit Online-Praktika an der Universitätsbibliothek Wien. In: Mitteilungen der Vereinigung Österreichischer Bibliothekarinnen und Bibliothekare 73.3-4 (2021), S. 422-434, https:// doi.org/10.31263/voebm.v73i3-4.5371.

Kobert, Elizabeth: Outlying Internships: Managing Remote Practicums and Internships During COVID-19, https://www.libraryjournal.com/?detailStory=0utlying-Internships-ManagingRemote-Practicums-and-Internships-During-COVID-19 [Zugriff: 15.05.2021].

Kole de Peralta, Kathleen: Curating COVID-19: A Digital Internship in a Rapid Response Archive. In: Collections: A Journal for Museum and Archives Professionals 1-10 (2020), https://doi. org/10.1177/1550190620981035.

Marczuk, Anna; Multrus, Frank; Lörz, Markus: Die Studiensituation in der Corona-Pandemie. Auswirkungen der Digitalisierung auf die Lern- und Kontaktsituation von Studierenden. Hannover 2021, https://doi.org/10.34878/2021.01.dzhw_brief.

Sarcletti, Andreas: Die Bedeutung von Praktika und studentischen Erwerbstätigkeiten für den Berufseinstieg. München 2009, https://www.ihf.bayern.de/uploads/media/ihf_studien_ hochschulforschung-77.pdf [Zugriff: 15.05.2021].

Utrecht Data School: Embedded Research Assistant, https://dataschool.nl/en/research/ embedded-research-assistant [Zugriff: 15.05.2021].

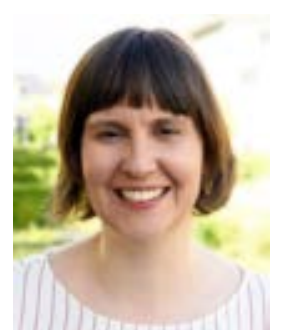

\title{
Prof. Dr. Elke Greifeneder
}

Humboldt-Universität zu Berlin

Institut für Bibliotheks- und Informationswissenschaft

Dorotheenstr. 26

10117 Berlin

Deutschland

E-Mail: greifeneder@ibi.hu-berlin.de

ORCID-ID: https://orcid.org/0000-0002-6814-3302

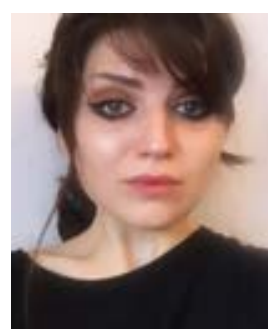

\author{
Adeline Krems \\ Humboldt-Universität zu Berlin \\ Institut für Bibliotheks- und Informationswissenschaft \\ Dorotheenstr. 26 \\ 10117 Berlin \\ Deutschland \\ E-Mail: adeline.krems@hu-berlin.de
}




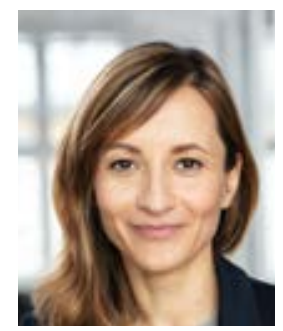

\section{Dr. Maria Gäde}

Humboldt-Universität zu Berlin

Institut für Bibliotheks- und Informationswissenschaft

Dorotheenstr. 26

10117 Berlin

Deutschland

E-Mail: maria.gaede@ibi.hu-berlin.de

ORCID-ID: https://orcid.org/0000-0002-1728-9440 